\title{
Quantum Theory of a Radiating Harmonically Bound Charge
}

\author{
Emilio Fiordilino \\ CNISM and Dipartimento di Scienze Fisiche e Astronomiche Università di Palermo, \\ Via Archirafi36, 90123Palermo, Italy \\ E-mail: fiordili@fisica.unipa.it
}

Received July 8, 2010; revised August 6, 2010; accepted August 18, 2010

\begin{abstract}
A phenomenological Hamiltonian giving the equation of motion of a non relativistic charges that accelerates and radiates is quantized. The theory is applied to the harmonic oscillator. To derive the decay time the physical parameters entering the calculations are obtained from the theory of the hydrogen atom; the agreement of the predicted value with the experiments is striking although the mathematics is very simple.
\end{abstract}

Keywords: Radiation Damping, Quantum Radiation, Phenomenological Hamiltonian

\section{Introduction}

A classical particle with charge $q$ and mass $m$, moving non relativistically with acceleration $\mathbf{a}(t)$, emits electromagnetic radiation whose power is given by the Larmor formula:

$$
P(t)=\frac{2}{3} \frac{q^{2}}{c^{3}}|\mathbf{a}(t)|^{2} .
$$

The equation of motion of the charge is modified by the loss of energy and assumes a form that is an open question and object of debate. The traditional non relativistic equation of motion for the charge moving in the presence of an external force $\mathbf{F}(\mathbf{r}, t)$ has the non newtonian form:

$$
m \frac{d^{2} \mathbf{r}}{d t^{2}}=\mathbf{F}(\mathbf{r}, t)+m \tau \frac{d^{3} \mathbf{r}}{d t^{3}}
$$

where $\tau=2 q^{2} /\left(3 m c^{3}\right)$ is $2 / 3$ of the time taken by the light to travel trough the classical radius of the charge; for the electron its value is $\tau=0.63 \times 10^{-23} \mathrm{sec}$. The equation of motion (2) was already known to H. A. Lorentz at the beginning of the XX century but it was P. A. M. Dirac [1] who gave a relativistically invariant method to derive the radiation reaction of which Equation (2) is the non-relativistic limit. A different approach taking to the same equation can be found in [2].

The standard solution of (2) requires three initial conditions and presents many problems of interpretation; if the force is function of the time alone, the acceleration obtained by solving Equation (2) is:

$$
\begin{aligned}
& \frac{d^{2} \mathbf{r}(t)}{d t^{2}}=\exp \left(\frac{t}{\tau}\right)\left\{\mathbf{a}\left(t_{0}\right) \exp \left(-\frac{t_{0}}{\tau}\right)\right. \\
& \left.-\frac{1}{m \tau} \int_{t_{0}}^{t} \exp \left(-\frac{t_{1}}{\tau}\right) \mathbf{F}\left(t_{1}\right) d t_{1}\right\}
\end{aligned}
$$

here $\mathbf{a}\left(t_{0}\right)$ is an integration constant which one is naturally pushed to identify with the initial acceleration at time $t_{0}$. Unfortunately by doing so $\ddot{\mathbf{r}}(t)$ diverges for large values of the time even when the external force has been switched off. Dirac [1] showed that the difficulty can be circumvented by requiring that the final acceleration is zero. By following this idea the solution of the equation of the motion is:

$$
\mathbf{a}(\mathbf{r}, t)=\frac{1}{m \tau} \int_{t}^{\infty} e^{\left(t-t_{1}\right) / \tau} \mathbf{F}\left(\mathbf{r}, t_{1}\right) d t_{1}
$$

which does not diverge but causes the particle to interact with the future; the final effect is that the charge begins to accelerate the tiny time $\tau$ before the onset of the force [3].

Exploiting the fact that $\tau$ is very small, it is possible to substitute in Equation (2) the quantity $\dot{\mathbf{F}}(\mathbf{r}, t)$ to $m \dot{\mathbf{a}}(t)$; in this way the equation of motion becomes

$$
m \mathbf{a}=\mathbf{F}+\tau \frac{d \mathbf{F}}{d t}=\mathbf{F}+\tau\left[\frac{\partial \mathbf{F}}{\partial t}+(\mathbf{v} \cdot \nabla) \mathbf{F}\right]
$$

that has a newtonian form and does not present exotic solutions; it can be considered an approximation of (2) 
for

$$
\left|\tau \frac{d \mathbf{F}}{d t}\right| \ll|\mathbf{F}|
$$

Equation (5) has been considered by Rohrlich in [4] to discuss the secular question of the radiation of an uniformly accelerated charge. Di Piazza in [5] has used its relativistic generalization to find the motion of a charge in the presence of a plane electromagnetic wave.

Let us consider now a charge acted upon by a one dimensional harmonic force $F$ with free angular frequency $\Omega$ so that $F=-m \Omega^{2} x$; in this case Equation (5) gives the equation of a damped harmonic oscillator with damping constant $\gamma=\tau \Omega^{2}$ :

$$
\ddot{x}+\tau \Omega^{2} \dot{x}+\Omega^{2} x=0
$$

with solution

$$
x=\exp \left\{-\frac{\gamma}{2} t\right\}\left[x_{s} \sin (\tilde{\Omega} t)+x_{c} \cos (\tilde{\Omega} t)\right]
$$

where $x_{s}$ and $x_{c}$ are integration constants and

$$
\tilde{\Omega}=\sqrt{\Omega^{2}-\frac{\gamma^{2}}{4}} \simeq \Omega-\frac{\gamma^{2}}{8 \Omega}
$$

is the angular frequency of oscillation of the damped oscillator and shows that the free angular frequency is shifted of the quantity $\Delta \Omega=\tau^{2} \Omega^{3} / 8$.

It is not surprising that the theory of a radiating charge is much better understood in the quantum realm where the radiation is the result of spontaneous transitions between stationary states induced by the interaction of the charge with the vacuum radiation field and where the frequency Lamb shift results from emission and absorption of a photon by the oscillator [6,7]. At the moment the quantum electrodynamics is the theory that best agrees with experiments and the only coherent way to treat the radiation from charges; the price to be payed is that the theory is not easily applied so that physical problems are usually modelled by forgetting completely the radiation reaction even when this should be present at least in principle. Generally speaking this is a good assumption, however.

Recently the development of high power lasers opened the channel to so called laser induced effects. Among these the process of high order harmonic generation is relevant for our discussion. Here atoms in the presence of a laser field of frequency $\omega_{L}$ become source of new electromagnetic radiation whose spectrum is formed by a large plateau of odd harmonics of $\omega_{L}$. The maximum detected harmonic order can be as large as 300 [8]. The radiation origins from the strong acceleration induced by the laser upon the charges of the atom. By assuming that only one electron is active, the emitted power is given by the Larmor formula with $\mathbf{a}(t)$ the quantum averaged electron acceleration:

$$
\mathbf{a}(t)=\frac{d^{2}}{d t^{2}}\langle\psi(\mathbf{r}, t)|\mathbf{r}| \psi(\mathbf{r}, t)\rangle
$$

and $\psi(\mathbf{r}, t)$ the wave function of the electron in the presence of the laser. Here again the radiative reaction is never taken into account even in principle.

This paper approaches the problem of the quantum theory of a radiating charge from a phenomenological point of view; the lack of first principles ground of the treatment will be justified by the simplicity of the mathematical machinery and by the results. The kernel of the idea resides in the quantization of a hamiltonian that gives the correct form of the classical equation of motion. Unfortunately there is not any hamiltonian that gives Equation (2) but it is possible to construct one for Equation (7). This paper applies the idea and shows that the method provides correct quantitative predictions.

\section{Theory}

The Hamilton equations applied to the classical hamiltonian:

$$
H=e^{-\gamma t} \frac{p^{2}}{2 m}+e^{\gamma t} \frac{1}{2} m \Omega^{2} x^{2}
$$

give the expressions:

$$
\dot{p}=-\frac{\partial H}{\partial x}=-e^{\gamma t} \mu \Omega^{2} x
$$

for the time derivative of the canonical momentum and

$$
\dot{x}=\frac{\partial H}{\partial p}=e^{-\gamma t} \frac{p}{m}
$$

for the velocity; the two combined expressions give the equation of motion of a damped harmonic oscillator:

$$
\ddot{x}+\gamma \dot{x}+\Omega^{2} x=0 ;
$$

after comparison with Equation (7) we obtain:

$$
\gamma=\tau \Omega^{2} \text {. }
$$

From the expression for the velocity (13) we see that the kinetic momentum $m \dot{x}$ differs from the canonical momentum $p$ : the quantity $p^{2} / 2 m$ cannot be given the meaning of kinetic energy; indeed the expression for the total mechanical energy is:

$$
E=\frac{1}{2} m(\dot{x})^{2}+\frac{1}{2} m \Omega^{2} x^{2}=e^{-\gamma t} H
$$

showing that $H$ cannot be identified with the energy of the oscillator. This is not new in the domain of electrodynamics where the Hamiltonian of a charge $q$ interact- 
ing with an electromagnetic field with potentials $\mathbf{A}(\mathbf{r}, t)$ and $\Phi(\mathbf{r}, t)$ is

$$
\mathcal{H}=\frac{1}{2 m}\left[\mathbf{p}-\frac{q}{c} \mathbf{A}(\mathbf{r}, t)\right]^{2}+\Phi(\mathbf{r}, t)
$$

giving for the kinetic momentum $m \dot{\mathbf{r}}=\mathbf{p}-(q / c) \mathbf{A}$ so that $\mathbf{p}^{2} / 2 m$ is not the kinetic energy.

Quantum mechanics has a general quantization recipe: in the hamiltonian substitute classical quantities $Q$ with operators $\hat{Q}$; therefore the hamiltonian (11) assumes a quantum role after the substitutions $H \rightarrow \hat{H}$ and $p \rightarrow \hat{p}=-i \hbar \partial_{x}$.

The exact solution of the related time dependent Schroedinger equation

$$
i \hbar \frac{\partial \psi(x, t)}{\partial t}=\hat{H} \psi(x, t)
$$

is thoroughly derived in [9] and subsequently applied to the problem of the coupling of a spin to the modes of a cavity [10]; the interested reader is therefore referred to these works. The important point is that the exact quantum solution displays an oscillating solution with free angular frequency given precisely by $\tilde{\Omega}$; therefore an equivalent of the Lamb shift is obtained also within this phenomenological treatment.

Here, to explore the potentiality of the method, we are interested in an approximated solution which exploits the smallness of the damping factor. If

$$
\hat{H}_{0}=\frac{\hat{p}^{2}}{2 m}+\frac{1}{2} m \Omega^{2} x^{2}
$$

denotes the formal quantum hamiltonian of the non radiating oscillator the hamiltonian (11) can be written as

$$
\begin{aligned}
& \hat{H}=e^{-\gamma t}\left(\hat{H}_{0}-\frac{1}{2} m \Omega^{2} x^{2}\right)+e^{\gamma t} \frac{1}{2} m \Omega^{2} x^{2} \\
& =e^{-\gamma t} \hat{H}_{0}+m \Omega^{2} \sinh (\gamma t) x^{2} .
\end{aligned}
$$

Again neither $\hat{H}$ nor $\hat{H}_{0}$ can be considered energy operator since $\hat{p}$ is not the kinetic momentum.

Since $\gamma$ is small we can Taylor expand $\hat{H}$ : $(\sinh (\gamma t) \cong \gamma t \ll 1)$

$$
\hat{H} \cong\left(1-\tau \Omega^{2} t\right) \hat{H}_{0}+m \Omega^{4} \tau t x^{2}
$$

and solve the Schroedinger equation with $\gamma=\Omega^{2} \tau$ as perturbation parameter. We set:

$$
\left.\psi(x, t)=\sum_{n=0}^{\infty} a_{n} e^{-i g_{n}(t)} \mid n\right)
$$

and substitute it in the time dependent Schroedinger equation. Usual projection procedure gives:

$$
\begin{aligned}
& i \hbar\left(\dot{a}_{\ell}-i \dot{g}_{\ell} a_{\ell}\right) e^{-i g_{\ell}(t)}= \\
& \left\{\hbar \omega_{\ell}+\Omega^{2} \tau\left[m \Omega^{2}\left(x^{2}\right)_{\ell \ell}-\hbar \omega_{\ell}\right] t\right\} a_{\ell} e^{-i g_{\ell}(t)} \\
& +m \Omega^{4} \tau t\left(x^{2}\right)_{\ell, \ell-2} a_{\ell-2} e^{-i g_{\ell-2}(t)} \\
& +m \Omega^{4} \tau t\left(x^{2}\right)_{\ell, \ell+2} a_{\ell+2} e^{-i g_{\ell+2}(t)}
\end{aligned}
$$

with $\left.\left.\left.\hat{H}_{0} \mid n\right)=\hbar \omega_{n} \mid n\right)=\left(n+\frac{1}{2}\right) \hbar \Omega \mid n\right)$ and

$$
\begin{aligned}
& \left(x^{2}\right)_{k n} \equiv\left(k\left|x^{2}\right| n\right) \\
& =\frac{\hbar}{m \Omega}\left\{\frac{\sqrt{(n-1) n}}{2} \delta_{k, n-2}+\right. \\
& \left.\left(n+\frac{1}{2}\right) \delta_{k, n}+\frac{\sqrt{(n+1)(n+2)}}{2} \delta_{k, n+2}\right\} ;
\end{aligned}
$$

we stress that $\hbar \omega_{n}$ cannot be interpreted as the energy of the eigenstate $\mid n$ ) since $\hat{H}_{0}$ is not the energy operator. The system of equations in (23) is readily solved:

$$
g_{\ell}(t)=\left(\ell+\frac{1}{2}\right) \Omega t
$$

and

$$
\begin{aligned}
& i \hbar \dot{a}_{\ell}=m \Omega^{4} \tau t\left[\left(x^{2}\right)_{\ell, \ell-2} a_{\ell-2} e^{i 2 \Omega t}\right. \\
& \left.+\left(x^{2}\right)_{\ell, \ell+2} a_{\ell+2} e^{-i 2 \Omega t}\right] .
\end{aligned}
$$

The system is solved with the initial condition $a_{\ell}(t=0)=a_{\ell} \delta_{\ell, K}$. First order perturbation theory reduces the system of equations to:

$$
\left\{\begin{array}{c}
i \hbar \dot{a}_{K+2}^{(0)}=\frac{\hbar \tau \Omega^{3}}{2} \sqrt{(K+1)(K+2)} e^{i 2 \Omega t} t \\
i \hbar \dot{a}_{K-2}^{(0)}=\frac{\hbar \tau \Omega^{3}}{2} \sqrt{(K-1) K} e^{-i 2 \Omega t} t
\end{array}\right.
$$

which after integration gives:

$$
\left\{\begin{aligned}
a_{K+2}^{(0)} & =\frac{\tau \Omega^{2}}{4} \sqrt{(K+1)(K+2)} \\
& \cdot\left\{-t e^{i 2 \Omega t}+\frac{e^{i \Omega t}}{\Omega} \sin \Omega t\right\} \\
a_{K-2}^{(0)} & =-\frac{\tau \Omega^{2}}{4} \sqrt{(K-1) K} \\
& \cdot\left\{-t e^{-i 2 \Omega t}+\frac{e^{-i \Omega t}}{\Omega} \sin \Omega t\right\}
\end{aligned}\right.
$$

correct for $t \ll \frac{1}{\Omega^{2} \tau}$. 


\subsection{Estimations}

The transition probabilities are now worked out:

$$
\begin{aligned}
& \left|a_{K+2}^{(0)}\right|^{2}=\frac{\tau^{2} \Omega^{2}}{2^{4}}(K+1)(K+2) \\
& \cdot\left\{\Omega^{2} t^{2}+\sin ^{2} \Omega t-\Omega t \sin (2 \Omega t)\right\} \\
& \left|a_{K-2}^{(0)}\right|^{2}=\frac{\tau^{2} \Omega^{2}}{2^{4}}(K-1) K \\
& \cdot\left\{\Omega^{2} t^{2}+\sin ^{2} \Omega t-\Omega t \sin (2 \Omega t)\right\}
\end{aligned}
$$

having in mind the hydrogen atom in the first excited state we set

$$
\begin{aligned}
& \Omega=\frac{1}{\hbar}\left(E_{2}-E_{1}\right)=\frac{3}{2^{3}} \frac{m e^{4}}{\hbar^{3}} . \\
& \psi_{K}(x, t)=e^{-i\left(K+\frac{1}{2}\right) \Omega t}\left\{|K\rangle+\frac{\tau \Omega^{2}}{4}\left[\sqrt{(K+1)(K+2)}\left(-t+\frac{e^{-i \Omega t}}{\Omega} \sin \Omega t\right)|K+2\rangle\right.\right. \\
& \left.\left.-\sqrt{(K-1) K}\left(-t+\frac{e^{i \Omega t}}{\Omega} \sin \Omega t\right)|K-2\rangle\right]\right\} \text {, } \\
& \hat{H} \psi_{K}(x, t)=e^{-i\left(K+\frac{1}{2}\right) \Omega t}\left(1-\tau \Omega^{2} t\right) \hbar \Omega\left\{\left(K+\frac{1}{2}\right)|K\rangle\right. \\
& +\frac{\tau \Omega^{2}}{4}\left[\sqrt{(K+1)(K+2)}\left(-t+\frac{e^{-i \Omega t}}{\Omega} \sin \Omega t\right)\left(K+2+\frac{1}{2}\right)|K+2\rangle\right. \\
& \left.\left.-\sqrt{(K-1) K}\left(-t+\frac{e^{i \Omega t}}{\Omega} \sin \Omega t\right)\left(K-2+\frac{1}{2}\right)|K-2\rangle\right]\right\} \\
& +m \Omega^{4} \tau t x^{2} e^{-i\left(K+\frac{1}{2}\right) \Omega t}\left\{|K\rangle+\frac{\tau \Omega^{2}}{4}\left[\sqrt{(K+1)(K+2)}\left(-t+\frac{e^{-i \Omega t}}{\Omega} \sin \Omega t\right)|K+2\rangle\right.\right. \\
& \left.\left.-\sqrt{(K-1) K}\left(-t+\frac{e^{i \Omega t}}{\Omega} \sin \Omega t\right)|K-2\rangle\right]\right\} \text {. }
\end{aligned}
$$

To first order in $\gamma$ :

$$
\langle\hat{H}\rangle \equiv \int_{-\infty}^{\infty} \psi_{K}^{*}(x, t) \hat{H} \psi_{K}(x, t) d x=\left(K+\frac{1}{2}\right) \hbar \Omega-\hbar \tau \Omega^{2} t\left(K+\frac{1}{2}\right) \Omega+m \Omega^{4} \tau t\left(x^{2}\right)_{K, K}=\left(K+\frac{1}{2}\right) \hbar \Omega .
$$

Our previous discussion on the energy of the radiating oscillator (16) shows that the energy decays as $E(t)=e^{-\gamma t}\langle\hat{H}\rangle \simeq(1-\gamma t)\langle\hat{H}\rangle$ as it should be.

\section{Discussion}

Hamiltonians of the type of formula (11) have been in the past applied to study the quantum counterpart of a particle in the presence of a mechanical dissipative force; the conclusion has often been negative: the quantum the- ory of particles moving in the presence of dissipative force is not yet complete. In [11] furthermore the very basic meaning of the mechanical system described by the hamiltonian (11) is questioned. In [12,13] an approach very similar to the one outlined in this work has been presented with purpose to study the somewhat artificial problem of quantum particles in the presence of a friction. The present work considers the radiation process as creating a dissipative medium and finds its frame in the domain of phenomenological quantum electrodynamics 
and not in the realm of mechanics.

The theory here outlined gives a practical, albeit ad hoc, method of incorporating the radiation reaction in the quantum treatment of a harmonically bound charge. Recently [14] it has been shown that the nuclei of a diatomic molecule driven by a laser field can be profitably described by assuming that they are bound by a harmonic potential so that such a system might provide a benchmark for the theory here presented.

\section{Acknowledgements}

This work has been supported by the Italian Ministero dell'Istruzione, dell'Università e della Ricerca and has been outlined while visiting A. Maquet in Paris. Discussions with A. Di Piazza, M. H. Mittleman and F. Persico are gratefully acknowledged.

\section{References}

[1] P. A. M. Dirac, "Classical Theory of Radiating Electrons," Proceedings of the Royal Society A, Vol. 167, No. 929, 1938, pp. 148-169.

[2] J. A. Wheeler and R. P. Feynman, "Interaction with the Absorber as the Mechanism of Radiation," Reviews of Modern Physics, Vol. 17, No. 2-3, 1945, pp. 157-181

[3] G. N. Plass, “Classical Electrodynamic Equations of Motion with Radiative Reaction,” Reviews of Modern Physics, Vol. 33, No. 1, 1961, pp. 37-62.

[4] F. Rohrlich, "Dynamics of a Charged Particle,” Physical Review E, Vol. 77, No. 4, 2008, p. 46609.

[5] A. D. Piazza, "Exact Solution of the Landau-Lifshitz Equation in a Plane Wave,” Letters in Mathematic Physics, Vol. 83, No. 3, 2008, pp. 305-313.

[6] P. W. Milonni, J. R. Ackerhalt and W. A. Smith, "Interpretation of Radiative Corrections in Spontaneous Emission,” Physical Review Letters, Vol. 31, No. 15, 1973, p. 958.

[7] J. J. Sakurai, “Advanced Quantum Mechanics,” AddisonWesley, Reading, London, 1982.

[8] Z. Chang, A. Rundquist, H. Wang, M. M. Murnane and H. C. Kapteyn, "Generation of Coherent Soft X Rays at 2.7 nm Using High Harmonics,” Physical Review Letters, Vol. 79, No. 16, 1997, pp. 2967-2970.

[9] K. W. H. Stevens, "The Wave Mechanical Damped Harmonic Oscillator," Proceedings of Physical Society, Vol. 72, No. 6, 1958, p. 1027.

[10] K. W. H. Stevens and B. Josephson Jr, "The Coupling of a Spin System to a Cavity Mode," Proceedings of Physical Society, Vol. 74, No. 5, 1959, p. 561.

[11] J. R. Ray, "Lagrangians and Systems they Describe-How Not to Treat Dissipation in Quantum Mechanics,” American Journal of Physics, Vol. 47, No. 7, 1979, p. 626.

[12] V. W. Myers, "Quantum Mechanical Treatment of Systems with a Damping Force Proportional to the Velocity," American Journal of Physics, Vol. 27, No. 7, 1959, pp. 507-508.

[13] L. H. Buch and H. H. Denman, "Solution of the Schrödinger Equation for Some Electric Problems,” American Journal of Physics, Vol. 42, No. 4, 1974, pp. 304-309.

[14] P. P. Corso, E. Fiordilino and F. Persico, "Direct Theoretical Evidence of Nuclear Motion in $\mathrm{H}_{2}^{+}$by Means of High Harmonic Generation,” Journal of Physics B: Atomic. Molecular and Optical Physics, Vol. 40, No. 7, 2007, p. 1383. 